\title{
Understanding Neutrino Oscillations with Particle Accelerator Experiments
}

\author{
Jiwon Woo ${ }^{1}$ and Gyuhyeon Lee ${ }^{1 \#}$ \\ ${ }^{1}$ Hankuk Academy of Foreign Studies, Yongin, Kyunggi, Korea \\ \#Advisor
}

ABSTRACT

Matter-dominant universe cannot be explained with the Standard Model. In order to understand why the current universe mainly consists of matter particles, scientists turned their attention to neutrino oscillations, and conducted research on the properties of the particle and its potential relationship with the matter-antimatter asymmetry observed in the universe. In this research, the probability function of a neutrino oscillation was studied for 2-neutrino case to understand neutrino oscillation in particle accelerator experiments. For a more practical study, the neutrino oscillation probability function was calculated for two neutrino experiments and was used to verify neutrino detector positions and calculated $\Delta \mathrm{m} 2$ which is mass difference between oscillating two different neutrinos. From this work, it was understood that detectors are located at positions with the highest probability for detecting neutrino oscillations, and it was also confirmed that neutrino were oscillating from muon neutrinos to electron neutrinos in particle accelerator experiments.

\section{Introduction}

The observable universe today is filled with matter particles, substances that were formed by the Big Bang and interacted with each other during the 13.8 billion years after the birth of the universe. In its infancy, the universe was a mixture of energy and elemental particles undergoing pair production and pair annihilation. From this mixture, the basic particles that compose today's universe were created. What is intriguing is the fact that, according to the Standard Model, an equal number of matter particles and antimatter particles should have been created during the pair production-annihilation phase. However, that is obviously not the case, as the observable universe is filled with matter. This problem of baryon asymmetry has baffled physicists and astronomers for decades, with scientists attempting to explain the phenomenon through research and experiment. Some of those experiments were to investigate whether the oscillation of neutrinos and antineutrinos violated CP-symmetry to a degree that it could explain the imbalance between the presence of matter and antimatter in the present universe.

In April of 2020, the researchers of T2K Collaboration released their results on their research of asymmetry in oscillations of neutrinos and antineutrinos.[1] Their results showed a high possibility of there being a difference in neutrino oscillation and antineutrino oscillation, with the research pointing to a likeliness of more electron neutrinos appearing compared to electron antineutrinos. This meant that there was a high chance of CP-symmetry being disrupted in neutrino oscillations, and that these neutrino oscillations could potentially be the answer to the question of why there were matter particles in abundance within the observable universe.

Based on this information, this research aimed to understand the reason behind conducting neutrino experiments such as $\mathrm{T} 2 \mathrm{~K}$, and how these experiments are attempting to prove that neutrino oscillations could be the reason that the universe is filled with matter today.

Matter-dominant Universe and Baryon Asymmetry 


\section{Matter and antimatter}

Shortly after the Big Bang and during the early stages of the universe, it is believed that through pair baryogenesis matter and antimatter particles were formed. These particles, formed from the immense heat and pressure caused by the Big Bang, annihilated in pairs, reverting themselves back into the form of energy. However, due to a mechanism that is yet to be discovered, there were slightly more matter particles than there were antimatter particles; it is estimated that for every billion pairs of particles annihilated, one matter particle survived.[2] As the universe expanded and the temperature decreased, the particles stopped their genesis and annihilation process. The remaining matter particles then slowly coalesced together to form massive clouds of stellar dust, becoming the seeds for forming the first generation of stars, galaxies, and ultimately the entire universe we see today.

\section{Baryon Asymmetry}

As mentioned above, the observable universe today is filled with matter. Quarks have coalesced to form protons and neutrons, which combined to form atomic nuclei. These atomic nuclei, along with electrons, formed atoms. These atoms compose interstellar objects, such as dust clouds, stars, planets, and asteroids. These celestial objects are combined by gravity to form bigger, larger structures such as galaxies, which are bound to their neighboring galaxies to form clusters. These clusters coalesce to form superclusters, which make up the backbone of the structure we see in the observable universe today.

The question remains, however, on how matter became so prevalent in the present universe. As mentioned in the previous unit, through baryogenesis, equal numbers of matter and antimatter particles ought to have been created. However, as evident from the state of the current universe, more matter particles than antimatter particles were created from the process; the excess matter particles that were left behind from the annihilation phase became the basis for the celestial objects observed today.
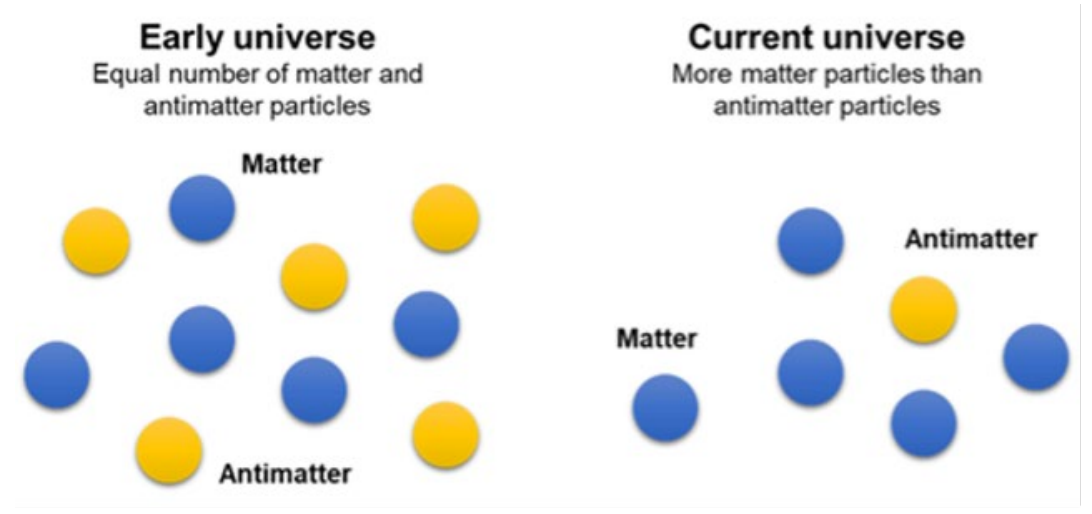

Figure 1. Baryon asymmetry explaining a difference between early universe and current universe. Early universe had equal numbers of matter and antimatter particles, but the current universe has more matter particles than antimatter particles

This seemingly paradoxical phenomenon, called baryon asymmetry or matter-antimatter asymmetry, has baffled many astronomers and physicists, and has led to the study of how more matter particles could have been formed compared to antimatter particles. Currently, research is ongoing in laboratories around the world, where researchers use particle accelerators to investigate possible mechanisms that would have caused the balance to tip. 


\section{Sakharov's Conditions for Baryon Asymmetry}

In 1967, in a research paper "Violation of CP-Invariance, C-Asymmetry, and Baryon Asymmetry of the Universe", Soviet nuclear physicist Andrei Sakharov proposed three conditions that would explain the seemingly contradictory baryon asymmetry: baryon number violation, violation of C-symmetry and CP-symmetry, and interactions out of thermal equilibrium.[3]

According to Sakharov, baryon number violation is necessary for baryon asymmetry to appear. Baryon number is defined through the formula $B=1 / 3$ (n_q-n_q ${ }^{-}$) where $B$ is the baryon number, $n \_q i s$ the number of quarks, and $n \_q$ - is the number of antiquarks. Within the Standard Model, the baryon number is considered to be conserved; that is, the sum of the baryon numbers of the incoming particles in a reaction must be equal to the sum of the baryon numbers of the resulting particles. However, in order to explain the discrepancy between the hypothetical amount of matter and antimatter particles and the amount observed in reality, the baryon number has to be violated in order to explain a mechanism that can disrupt the pair-baryogenesis and pair-annihilation.

Another condition is that both C-symmetry, short for charge symmetry, and CP-symmetry, short for charge conjugation parity symmetry, needs to be disrupted. Charge symmetry needs to be violated since charge symmetry means that for every type of baryon particle with a specific charge, there is an antimatter counterpart with the opposite charge. The same can be applied for CP-symmetry; CP-symmetry dictates that for every matter baryon particle with a specific helicity, there exists an antimatter baryon particle with the opposite helicity.

Finally, the violation of thermal equilibrium is required, as in order for the baryon asymmetry to continue, the universe has to expand faster than the rate of the reaction that creates the baryon asymmetry.

\section{Alternate Hypotheses for Baryon Asymmetry}

There are multiple attempts to use alternate theories to explain the baryon asymmetry. Several of them will be introduced in the following points made.

The first of these hypotheses is the Patchwork Universe. This hypothesis assumes that the Universe, in fact, is made up of separate regions that are either filled with matter or antimatter. The theory suggests that there are distinct regions within the universe, with regions filled with matter being separated from antimatter regions. According to "Matter and Antimatter in the Universe" by Laurent Canetti, et al., no antimatter particles or gamma rays consistent to those expected from the annihilation process occurring between matter regions and antimatter regions have been detected within the Milky Way, effectively eliminating the possibility that such regions exist on a galactic scale. However, the possibility remains that such regions are comparable to the Observable Universe in terms of size, separated by large voids devoid of baryon matter, based on observation data obtained from space observatories.[4]

Another hypothesis is related to the electric dipole moment of fundamental particles. If a fundamental particle possesses an electric dipole moment, the parity symmetry and the time symmetry of the particle is broken, causing a difference in the rate of decay between matter particles and their antimatter counterparts. This would cause a difference in the number of matter particles and antimatter particles, resulting in the matter-dominant universe seen today. For this purpose, research on the electric dipole moments of fundamental particles is ongoing, such as securing the limit of the electric dipole moment of an electron.[5]

Another alternate hypothesis is a Mirror Anti-Universe. According to "CPT-Symmetric Universe" by Lathan Boyle et al., a way to explain the phenomena observed in the Observable Universe was to assume a mirrored universe consisting of antimatter particles, growing in size as time flowed backward. This hypothesis explains the dark matter as well, suggesting that it consists of sterile neutrinos.[6]

\section{Neutrinos and Neutrino Oscillations}




\section{Definition and Properties of Neutrino Particles}

Neutrinos, which means "little neutral one" in Italian, were first proposed in 1930 by Wolfgang Pauli in order to explain the conservation of energy, momentum, and spin in the Beta decay, as opposed to the proposal made by Niels Bohr, who suggested a statistical version of the conservation laws. As a neutron decays into a proton, it emits an electron and an electron neutrino (see III-B for more on the type of neutrinos). As neutrinos were unknown at the time, Pauli could only postulate that a particle like that existed. At this time, Pauli called these particles "neutrons", as the much more massive neutral particle hadn't been discovered yet; the neutron was discovered by James Chadwick in 1932, after which Enrico Fermi dubbed the lighter particle "neutrino" in order to avoid confusion. In 1934 Fermi published a paper on his theory of beta decay, in which he explained how a neutron could decay into a proton, emitting an electron and a particle that is now called the electron antineutrino.[7] In 1942 it was proposed by Wang Ganchang to use the process of beta capture, or electron capture, to experimentally prove the existence of neutrinos. In 1956, in what is now known as the Cowan-Reines neutrino experiment, physicists Clyde L. Cowan and Fredrick Reines used a water-filled tank sandwiched between layers of scintillators, with cadmium chloride dissolved in water in order to increase the certainty of a neutrino detection. The two detected gamma rays as they had predicted, experimentally proving that neutrino was a real particle indeed.

As predicted by physicists prior to its discovery, the neutrino is a very light weight particle with no electric charge and interacts only with the gravitational and weak force. Since the particle is too light for gravity to have a significant effect on the particle, and since weak interactions have a very short range, most neutrinos pass through matter undisturbed.

Several properties of neutrinos include, but are not limited to:

having three types, or flavors, each named muon neutrino, tau neutrino, and electron neutrino.

oscillation between these three flavors, with each configuration of a neutrino being a mixture of the three flavors.

has a spin value of $1 / 2$, meaning that the particle is a fermion. There are multiple attempts to use alternate theories to explain the baryon asymmetry. Several of them will be introduced in the following points made.

\section{Types of Neutrinos and Neutrino Oscillations}

As mentioned earlier, there are three flavors in neutrinos. Dubbed muon neutrino, tau neutrino, and electron neutrino, each type is formed through different mechanisms. The electron neutrino was the first to be discovered, first postulated of its existence in 1930 and discovered in 1956 through the aforementioned Cowan-Reines neutrino experiment. The muon neutrino came next when it was discovered in 1962 from the results of a particle accelerator experiment, when pions decayed into muon neutrinos which were then sent to the detector to be detected. Then finally the tau neutrino followed, theorized in the mid-1970s as a particle associated with the tau particle before observing the direct experimental evidence to the tau neutrino's existence in 2000 through the DONUT experiment at Fermilab.

Neutrinos, as they travel through space, interact with surrounding matter as if it were a mixture of these three flavors. In other words, the state of these three flavors differs from the state observed in each flavored neutrino.

It has also been proved through many experiments that as neutrinos traverse through space they experience oscillations, resulting in the neutrino changing flavors by the time they arrive at the detector. For example, a neutrino that started as an electronic neutrino from a beta decay could be detected as a muon or tau neutrino when it arrives at the detector. This is thought to be caused by each flavor of the neutrino having nonzero and slightly different masses, which travel at different speeds as wave functions. As these masses travel through space in the form of waves, they experience interference with one another. Through this interference, the different flavors of neutrinos emerge at different times until the observation made by the detector causes the wave functions to collapse, fixing the flavor of the particle into one given state. 


$$
p\left(v_{1} \rightarrow v_{2}\right)=\sin ^{2}(2 \theta) \sin ^{2}\left(\frac{\Delta m^{2} L}{4 E_{v}}\right)=\sin ^{2}(2 \theta) \sin ^{2}\left(1.27 \frac{\Delta m^{2}[\mathrm{eV}] L[\mathrm{~km}]}{E_{v}[\mathrm{GeV}]}\right)
$$

Eq. (1) states the function for calculating the probability of neutrinos converting their flavor from their beginning status to another status in a two-neutrino problem based on distance. The $\sin ^{2}(2 \theta)$ in the equation defines the amplitude, or the maximum possibility of a conversion happening, while the $\Delta m^{2}$ is the difference of the square of the mass of the particles. $L$ states the distance the neutrino particles travel in kilometers and is the main variable of this function.

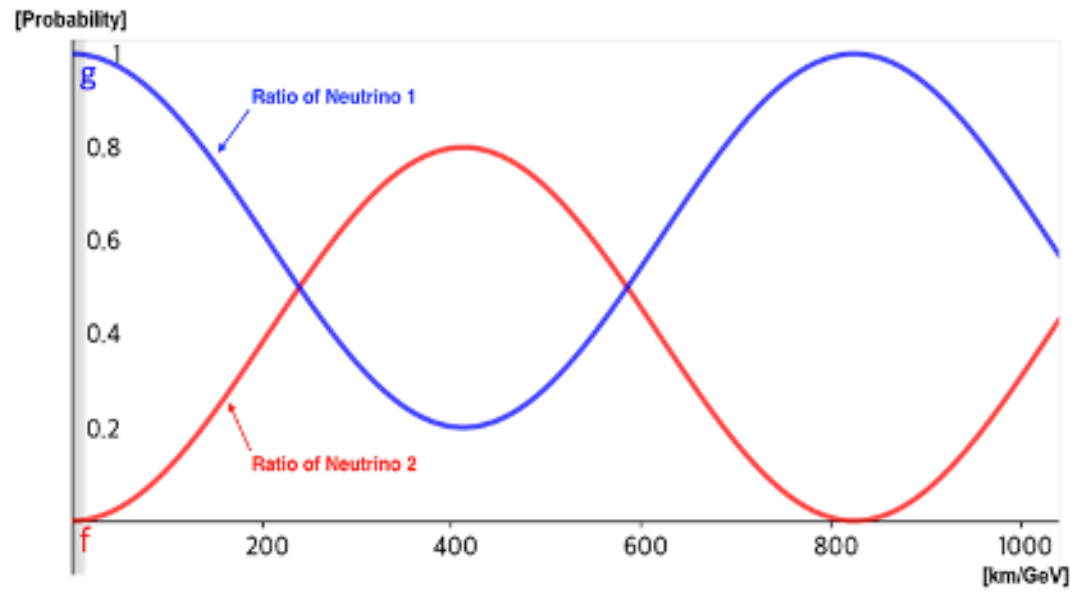

Figure 1. Neutrino oscillation between two different neutrinos. Probability of neutrino 1 is decreasing while probability of neutrino 2 is increasing when neutrino is traveling in a certain distance

A graphical rendition of the function with the values shown below substituting the various parameters; $m^{2}=0.003$ $\mathrm{eV}^{2}, \sin ^{2}(2 \theta)=0.8, E_{v}=1 \mathrm{GeV}$ in Eq. (1) is shown in Fig. 2, where the possibility of neutrino changing flavor is shown to oscillate in distance.

\section{Relationship between Neutrino Oscillations and Baryon Asymmetry}

As explained in Sakharov's Proposed Conditions for Baryon Asymmetry, it is hypothesized that three conditions need to be met in order for baryon asymmetry to occur; violation of baryon number, violation of C-symmetry and CPsymmetry, and the violation of thermal equilibrium.

In order to explain the discrepancy between the Standard Model and the observed unbalance between matter and antimatter particles in the current universe, scientists have been conducting experiments to uncover whether violation of CP-symmetry was observable in the formation and interactions of matter and antimatter particles. So far, the CPsymmetry violation observed in quarks has been insufficient to explain the difference in the ratio of naturally observed matter and antimatter particles. However, a discrepancy has been found in the neutrino/antineutrino oscillations, where scientists suspect that the rate of oscillations differ and thus creating a difference in the rate matter and antimatter are formed from interactions between basic particles, thus opening a possibility that the asymmetry in the rate of oscillations in neutrino and antineutrino could be the cause of the baryon asymmetry observed in the universe today. This led the scientists to conduct experiments with neutrino and antineutrino beams to investigate whether the rate of oscillations differ in the two types of the same particle.

\section{Various Neutrino Experiments}




\section{The Tokai to Kamioka (T2K) Experiment}

The Tokai to Kamioka Experiment, more commonly called the T2K Experiment, is an experiment for investigating the oscillations of neutrinos. Built $1 \mathrm{~km}$ underground in order to minimize outer neutrino interference, the Super Kamiokande detector contains 50000 tons of water, and has 13000 photomultiplier tubes installed. As a neutrino particle interacts with the water inside the detector, it causes Cherenkov radiation, a phenomenon where particles move faster inside a certain medium than the speed of light inside that medium. The photomultiplier tubes detect light from the Cherenkov radiation and analyze the patterns shown in the light emission. A muon neutrino interacts with particles to create muons, which in turn cause a ring-shaped Cherenkov light emission with well-defined edges. On the other hand, an electron neutrino creates relatively lighter electrons, which are more prone to scattering and thus create ring-shaped light emission with fuzzy edges. Using this principle, the T2K experiment uses either a muon neutrino beam or a muon antineutrino beam created in Tokai, which is then directed towards the Super Kamiokande detector $295 \mathrm{~km}$ away. The T2K experiment is designed to detect neutrinos at the low energy range of around 600 $\mathrm{MeV}$.

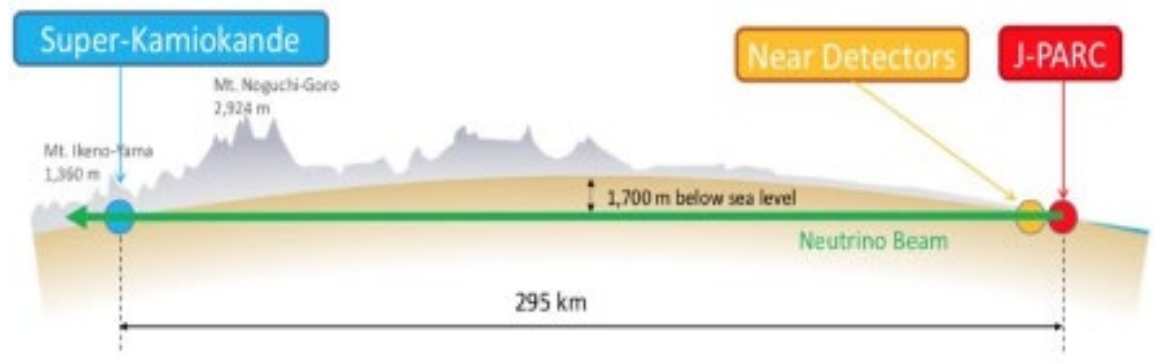

As mentioned earlier in the paper, a team of researchers at T2K announced in April that through T2K they had managed to exclude half of the possible values in the difference between neutrino oscillations and antineutrino oscillations, with statistics pointing to a high possibility that more electron neutrinos emerged than their antimatter counterparts as a result of neutrino/antineutrino oscillations. In this experiment, the team used the accelerator to launch $1.49 \times 10^{21}$ and $1.64 \times 10^{21}$ protons in neutrino beam mode and antineutrino beam mode, respectively. They then analyzed the observed neutrino detection events and determined the number of detected electron neutrinos and electron antineutrinos. Prior to the experiment, the researchers had predicted that they would detect 82 electron neutrinos and 17 electron antineutrinos for maximal neutrino enhancement $\left(\delta_{c p}=-90^{\circ}\right)$ and 56 electron neutrinos and 22 electron antineutrinos for maximal antineutrino enhancement $\left(\delta_{c p}=90^{\circ}\right)$. In the experiment, the researchers detected 90 electron neutrinos and 15 electron antineutrinos, proof that the conditions of the neutrino oscillations were closer to the conditions of maximal neutrino enhancement. The research team concluded that this result provided enough statistical evidence to exclude nearly half of the possible values for $\delta_{c p}$ with a confidence level of $3 \sigma$, with the exclusion eliminating most of the possibilities of an antineutrino enhancement. From this, the research team concluded that it was likely that CP-symmetry was violated in the neutrino oscillation, yet that conclusion could not be ascertained with a high enough confidence level. The research team at T2K planned to heighten the confidence level by upgrading the equipment and detectors for measurements in the near future.

\section{DUNE/LBNF}


The DUNE/LBNF is an experiment that is scheduled to be conducted in the United States, where a beam of neutrino particles will be launched from Fermilab to Deep Underground Neutrino Experiment at Sanford, South Dakota, 1300 $\mathrm{km}$ away from the neutrino beam source. The experiment is expected to bring a deeper understanding of the nature of the neutrino oscillation, as well as properties of neutrinos that are yet to be uncovered. Such properties include, but are not limited to, the existence of a sterile neutrino, the mass of the three flavors of neutrinos, and so on. The DUNE/LBNF experiment will be using the most intense neutrino beam to be used in a neutrino experiment which is expected to increase the precision of the experiments conducted. It is also expected to be able to adjust the energy level of the neutrino beams, which will help in investigating how neutrinos behave differently based on energy levels. The detectors will be placed $1.3 \mathrm{~km}$ underground, with 70000 tons of liquid argon filled into the detector tanks. The cold liquid argon is expected to enhance the detector's capabilities to capture the particle interactions occurring inside the detector in greater detail.

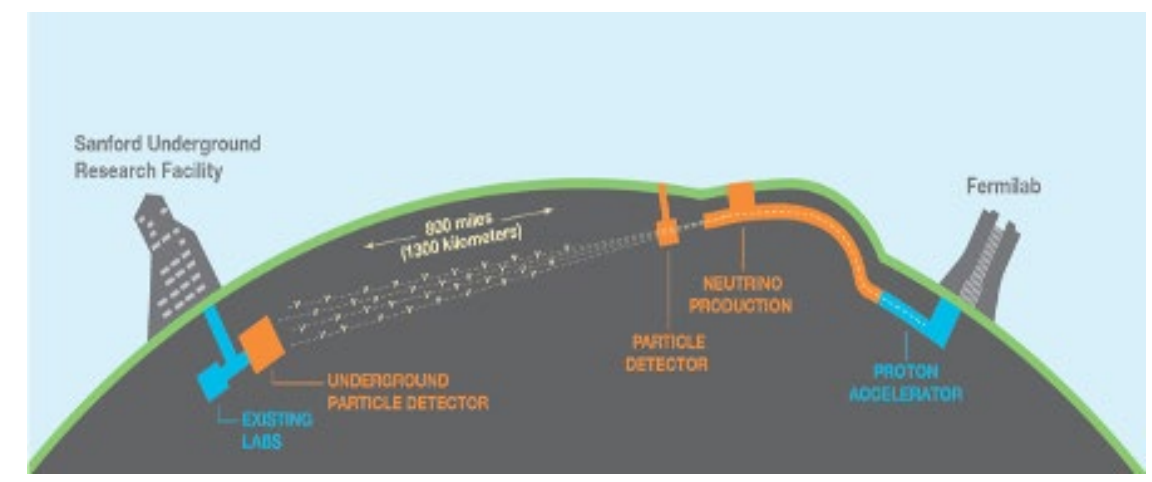

\section{Discussion}

\section{General Comparison of T2K and DUNE/LBNF}

In the previous chapter, characteristics and goals of two experiments, the $\mathrm{T} 2 \mathrm{~K}$ experiment and the planned DUNE/LBNF experiment, has been discussed. Both experiments aim to uncover the answers to the question of baryon asymmetry through finding CP-symmetry violation in neutrino oscillations. However, there are several differences in the two experiments. For one, the distance between the neutrino source and the far detector are very different; the distance from Tokai to Kamiokande is $295 \mathrm{~km}$, while the distance from Fermilab in Chicago to DUNE in South Dakota is $1300 \mathrm{~km}$. Another difference is the energy level used for the neutrino beams. T2K uses a relatively low energy neutrino beam of $600 \mathrm{MeV}$, while DUNE/LBNF is planning to use a higher energy neutrino beam of $1 \sim 5 \mathrm{GeV}$. One more major difference would be the detection medium used in the detectors of the two experiments. T2K uses 50000 tons of purified water, while DUNE/LBNF is planning to use 70000 tons of liquid argon. An organized comparison of these differences in the experimental factors is shown in Table 1.

Table 1. Comparison of particle accelerator experiments in T2K and DUNE/LBNF

\begin{tabular}{l|l|l}
\hline & T2K & DUNE/LBNF \\
\hline \hline $\begin{array}{l}\text { Distance between Neutrino Source } \\
\text { and Detector }\end{array}$ & $295 \mathrm{~km}$ & $1300 \mathrm{~km}$ \\
\hline
\end{tabular}




\begin{tabular}{l|l|l}
\hline Energy Level of Neutrino Beam & $600 \mathrm{MeV}$ & $1 \sim 5 \mathrm{GeV}$ \\
\hline Detection Medium & 50000 tons of Purified Water & 70000 tons of Liquid Argon \\
\hline $\begin{array}{l}\text { Value of } \Delta m^{2} \text { for Optimal } \\
\text { Observations }\end{array}$ & $2.51 \times 10^{-3} \mathrm{eV}^{2}$ & $2.85 \times 10^{-3} \mathrm{eV}^{2}$ \\
\hline
\end{tabular}

\section{The Locations of the Neutrino Detectors}

One of the experimental factors of interest is the two experiments' distance between their respective neutrino source and the far detector. As mentioned earlier, the distance from Fermilab to DUNE is more than 4 times the distance between Tokai and Kamiokande. It is thought that the reason for this is the difference in the energy level of the neutrino beams. As shown in Eq. (1), the oscillation of neutrinos based on distance depends on the energy level of the neutrino beam. The higher the energy level, the longer the distance traveled per oscillation probability cycle. Based on this, it can be concluded that the neutrinos from the DUNE/LBNF experiment would have to travel a longer distance in order to finish one probability cycle. As shown in Fig. 3 and 4, detector locations for T2K and DUNE experiments were set at the positions where neutrino changing ratio is the highest. This is because those positions are the best position for detecting neutrino oscillations.

\section{Calculation and Speculation of $\Delta \boldsymbol{m}^{2}$}

As shown in Table 1, the distance used in T2K is $295 \mathrm{~km}$, while the distance used in DUNE/LBNF is $1300 \mathrm{~km}$. As mentioned, assuming that the location of the detectors for each experiment is in the optimal location for detection of electron neutrinos, the value of $\Delta m^{2}$ can be calculated by substituting the variables in Eq. (1) with values that are already known.

Differentiating Equation 1 produces Eq. (2):

$$
\frac{d p}{d L}=\sin ^{2}(2 \theta) \times 2 \sin \left(1.27 \frac{\Delta m^{2} \cdot L}{E_{v}}\right) \times \cos \left(1.27 \frac{\Delta m^{2} \cdot L}{E_{v}}\right) \times 1.27 \frac{\Delta m^{2}}{E_{v}}=0
$$

Then, Eq. (2) becomes

$$
\cos \left(1.27 \frac{\Delta m^{2} \cdot L}{E_{v}}\right)=0
$$

For the T2K experiment, assuming that the value of $\sin ^{2}(2 \theta)$ is set to 0.8 like it was done in Fig. 1 and inserting $600 \mathrm{MeV}$ for the $E_{v}$ in the denominator of Eq. (3) produces a function based on $\Delta m^{2}$ and $L$, with the local extrema having to be at $L=295 \mathrm{~km}$. Calculating for $\Delta \mathrm{m}^{2}$ yields $2.52 \times 10^{-3} \mathrm{eV}^{2}$ as its value. Doing the same calculation for DUNE/LBNF yields the value $2.85 \times 10^{-3} \mathrm{eV}^{2}$ as the value of $\Delta \mathrm{m}^{2}$.

As seen from the calculations, there is a discrepancy in the values of $\Delta m^{2}$ derived from the assumption that the two detectors are placed in locations where the probability of observing neutrino oscillations is the highest.

Based on these calculations, a graphical rendition of the probability function of neutrino oscillations based on distance has been shown as Fig. 5 and Fig. 6. 


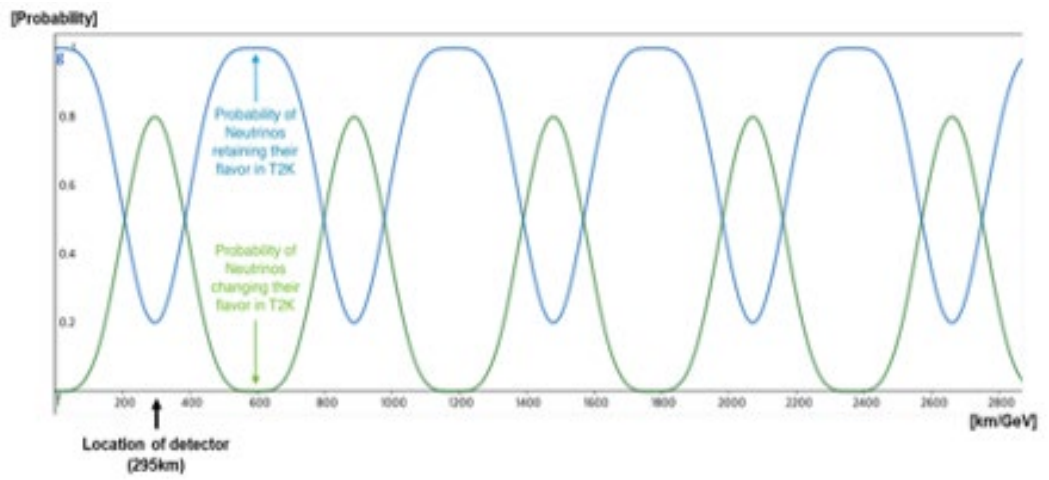

Figure 5. Probability of neutrino oscillations based on travel distance in T2K experiment. Maximum oscillation between neutrino 1 and neutrino 2 is happening at $295 \mathrm{~km}$

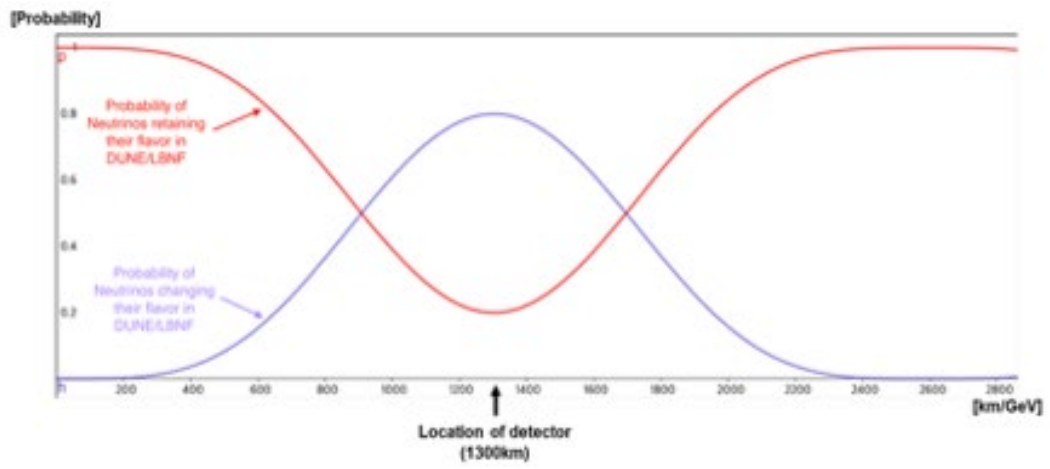

Figure 6. Probability of neutrino oscillations based on travel distance in DUNE/LBNF experiment. Maximum oscillation between neutrino 1 and neutrino 2 is happening at $1300 \mathrm{~km}$

The discrepancy of the calculated values of $\Delta m^{2}$ could be due to a number of factors. For example, the exact value of $\Delta m^{2}$ is unclear as of present, although it has been confirmed that it is somewhere within the range of $10^{-3} \mathrm{eV}^{2} . \mathrm{Also}$, the principal assumption behind the calculations was that the detectors were on the exact locations that guaranteed the optimal conditions for observing neutrino oscillations - this may not be true in reality, as there are other things to be considered when constructing a massive neutrino detection chamber.

As shown in Fig. 7, it has been experimentally shown that there are three mass states of neutrinos. These mass states each have a different probability of becoming unique flavors, with different energy levels for each mass state. These mass states are each referred to as $v_{1}, v_{2}, v_{3}$ with $v_{1}$ having a $2 / 3$ chance of interacting to become an electron neutrino, and $\mathrm{a} 1 / 3$ chance of becoming a muon or a tau neutrino. $v_{2}$ has roughly equal probability of becoming either of the three flavors. $v_{3}$ has a $45 \%$ probability of becoming a muon neutrino and a $45 \%$ probability of becoming a tau neutrino, and a $10 \%$ chance of becoming an electron neutrino. The difference between the energy levels for each mass state has been measured from various observations of interactions of neutrinos occurring in the Sun and Earth's atmosphere. Through this, the difference between $v_{1}$ and $v_{2}$, as well as the difference between $v_{2}$ and $v_{3}$ have been measured, from which it was concluded that the $\Delta m^{2}$ value for $v_{1}$ and $v_{2}$ was on the scale of $1 \times 10^{-5} \mathrm{eV}^{2}$, while the $\Delta \mathrm{m}^{2}$ value for $v_{2}$ and $v_{3}$ was estimated to be 400 times greater than the $\Delta m^{2}$ value for $v_{1}$ and $v_{2}$. From this, it can be deduced that the value of $\Delta m^{2}$ calculated earlier in this paper is the value of $\Delta m^{2}$ for the difference between either $v_{2}$ and $v_{3}$, or $v_{1}$ and $v_{3}$. This, combined with the information shown in Fig. 7, leads to the conclusion that it is likely that $v_{3}$ is very closely related to the mass of the muon neutrino, as the muon neutrino is the original state of neutrinos in the accelerator experiments mentioned earlier. 

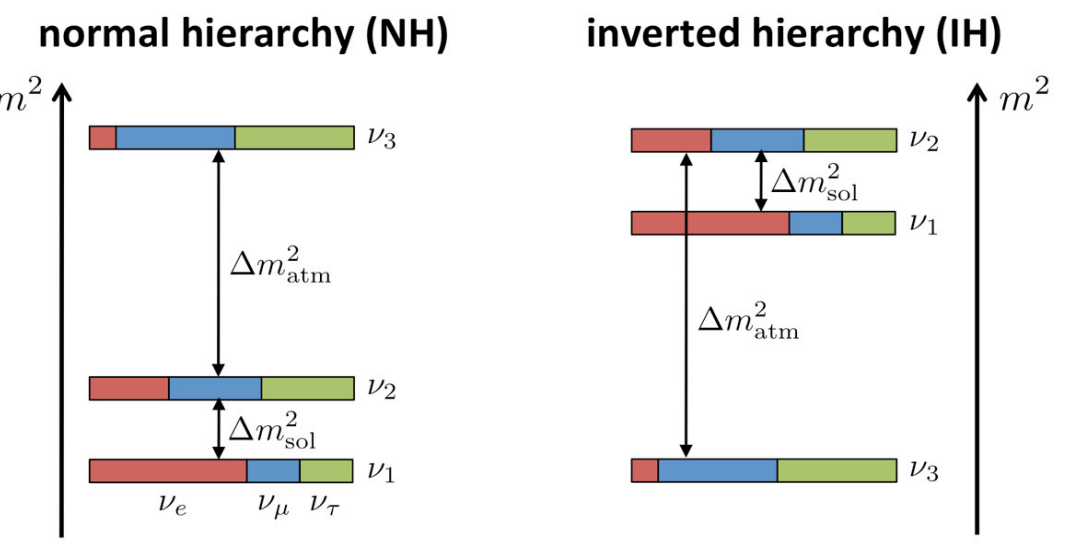

Figure 7. Hierarchy of Neutrino mass states and $\Delta m^{2}$ between different neutrinos. Each of three different neutrino phases (Muon, tau and electron neutrinos) has three different mass states[10]

\section{Conclusion}

This research began in order to understand how neutrino oscillations could be the answer to explaining baryon asymmetry. Through understanding other researches about neutrino oscillations and CP-symmetry violation, it was understood that by proving neutrino oscillations violate CP-symmetry, the problem of baryon asymmetry in the universe could be answered.

Then two major neutrino experiments, T2K and the currently-planned DUNE/LBNF, were compared to each other. Through this comparison, it was concluded that these two experiments placed their detectors in locations where the neutrino oscillation could be relatively easily detected with the highest oscillation probability. Based on this information, calculating the approximate $\Delta m^{2}$ was attempted, with the values derived from the distance between the neutrino beam source and the detector and the energy level of the beam showing the $\Delta m^{2}$ to be near the order of $10^{-3}$ $\mathrm{eV}^{2}$, although the two values were different from each other. This confirms that the neutrino oscillation detected in these experiments is from muon neutrinos to electron neutrinos which is designed for the experiments.

\section{Acknowledgments}

I specially thank Prof. Jaehoon Yu from University of Texas at Arlington. Prof. Yu supported this research by spending a lot of time to discuss in emails with me and kindly answered my questions on neutrinos experiments, accelerators and their background theories which provided me valuable knowledge and inspiration.

\section{References}

The T2K collaboration, "Constraint on the matter-antimatter symmetry-violating phase in neutrino oscillations", Nature, Volume 580, pp. 339-344 (2020)

CERN, "The matter-antimatter asymmetry problem", CERN, 2020, https://home.cern/science/physics/matter-antimatter-asymmetry-problem

A. D. Sakharov, "Violation of CP invariance, C asymmetry, and baryon asymmetry of the universe". Journal of 
Canetti, L. et al., "Matter and Antimatter in the Universe", New J. Phys., 14 (9), 095012 (2012)

The ACME Collaboration, "Order of Magnitude Smaller Limit on the Electric Dipole Moment of the Electron", Science, Vol. 343, Issue 6168, pp. 269-272 (2014)

Latham Boyle et al., “CPT-Symmetric Universe”, Phys. Rev. Lett. 121, 251301 (2018)

Fred L, Wilson, “Fermi’s theory of Beta decay”, American Journal of Physics, Vol. 26 (12), 1150-1160 (1968)

The T2K collaboration, “About T2K”, The T2K collaboration, 2020, https://t2k-experiment.org/t2k

Fermi National Accelerator Laboratory, “Deep Underground Neutrino Experiment”, Fermi Research Alliance, 2020, https://www.dunescience.org

Fermi National Accelerator Laboratory, “All things neutrino”, Fermi Research Alliance, 2020, https://neutrinos.fnal.gov 